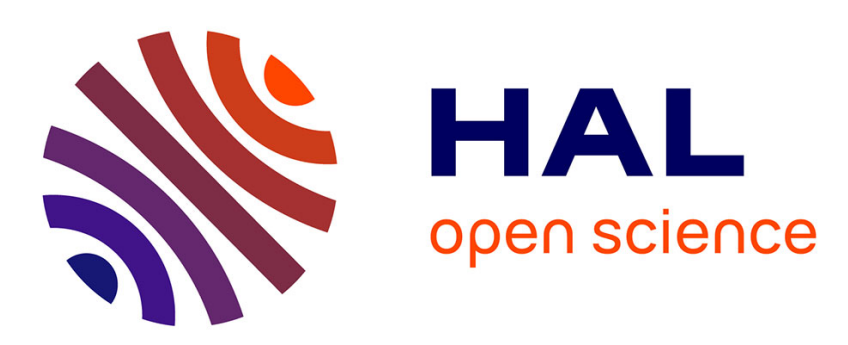

\title{
Vibrational Sum-Frequency Generation Spectroscopy in the Energy Representation from Dual-Level Molecular Dynamics Simulations
}

Marilia Martins-Costa, Manuel Ruiz-Lopez

\section{- To cite this version:}

Marilia Martins-Costa, Manuel Ruiz-Lopez. Vibrational Sum-Frequency Generation Spectroscopy in the Energy Representation from Dual-Level Molecular Dynamics Simulations. Journal of Physical Chemistry A, 2020, 124 (27), pp.5675-5683. 10.1021/acs.jpca.0c02901 . hal-03040040

\author{
HAL Id: hal-03040040 \\ https://hal.science/hal-03040040
}

Submitted on 4 Dec 2020

HAL is a multi-disciplinary open access archive for the deposit and dissemination of scientific research documents, whether they are published or not. The documents may come from teaching and research institutions in France or abroad, or from public or private research centers.
L'archive ouverte pluridisciplinaire HAL, est destinée au dépôt et à la diffusion de documents scientifiques de niveau recherche, publiés ou non, émanant des établissements d'enseignement et de recherche français ou étrangers, des laboratoires publics ou privés. 


\section{Vibrational Sum-Frequency-Generation}

\section{Spectroscopy in the Energy Representation from}

\section{Dual-Level Molecular Dynamics Simulations}

Marilia T. C. Martins-Costa ${ }^{\S}$ and Manuel F. Ruiz-López $z^{\S *}$

${ }^{\S}$ Laboratoire de Physique et Chimie Théoriques, UMR CNRS 7019, University of Lorraine, CNRS, BP 70239, 54506 Vandoeuvre-lès-Nancy, France

AUTHOR INFORMATION

Corresponding Author

*Manuel.Ruiz@univ-lorraine.fr 
ABSTRACT We report a cost-effective molecular dynamics approach to calculate sumfrequency-generation (SFG) vibrational spectra of molecular species at liquid interfaces in the energy representation formalism that brings together the instantaneous normal mode (INM) analysis at free energy minima (FEM) and the dual-level free-energy perturbation (FEP) methods. This combined FEP-INM-FEM approach allows analyzing SFG spectra in terms of normal mode contributions at very-high ab initio levels, in contrast to standard time-correlation function (TCF) based methods, from which it can be considered complementary. It is applied here to the study of the $\mathrm{CH}_{3}$-stretching band of methanol at the air-water interface, which has been thoroughly studied in the literature. The SFG band of acetonitrile in the same conditions has also been calculated with the aim of testing the capability of the method to reproduce small chemical shifts. The suitability of the proposed model is demonstrated by comparing the results with TCF data from QM/MM simulations, and with experiments. Analysis of these results provides new insights into the strength and orientational dependence of the SFG signal of symmetric and asymmetric stretching vibrations of $\mathrm{CH}_{3}$ groups, which can be of paramount importance to analyze the spectra of more complex systems. 


\section{TOC GRAPHICS}

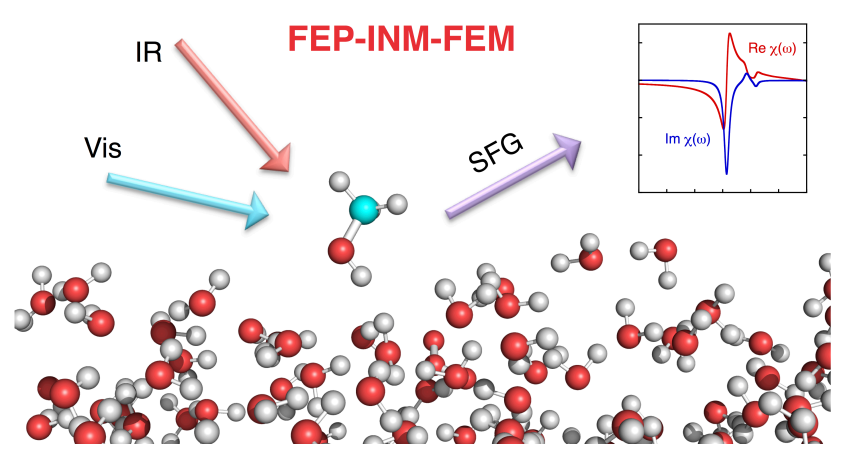

KEYWORDS SFG spectroscopy. Air-water interface. Molecular Dynamics. Correlation functions. Instantaneous Normal Modes. Methanol. Acetonitrile.

\section{Introduction}

Sum-Frequency Generation (SFG) vibrational spectroscopy has emerged in recent years as a powerful tool to analyze surfaces and interfaces.${ }^{1-4}$ In this technique, photons from a probing Visible beam interact with photons from an IR laser to produce photons with the sum of the two frequencies (SFG process). By tuning the IR laser frequency and monitoring the SFG intensity, the SFG-vibrational spectrum is obtained. For a vibrational transition to be observable in the SFG spectrum, it must be both IR and Raman-active. The SFG process is therefore forbidden in systems with a center of symmetry, which is the case for bulk solution but not for interfacial systems, where symmetry is broken. SFG vibrational spectroscopy allows the study of molecular dynamics at interfaces, e.g. through time-resolved or two-dimensional SFG techniques.-12 
Nevertheless, the analysis of experimental spectra is often a complicated task that can be greatly simplified by carrying out numerical simulations, and therefore any effort to develop more accurate methods is of utmost importance. The theory has been detailed in many reviews and we just summarize it here. The SFG intensity is proportional to the square of the effective non-linear surface susceptibility tensor $\chi_{\operatorname{lmn}}^{e f f}\left(\omega_{S F G}, \omega_{v i s}, \omega_{I R}\right)$ and to the intensities of the incident visible and IR beams: ${ }^{13-14}$

$I_{l}\left(\omega_{S F G}\right) \propto \omega_{S F G}^{2}\left|\chi_{l m n}^{e f f}\left(\omega_{S F G}, \omega_{v i s}, \omega_{I R}\right)\right|^{2} I_{m}\left(\omega_{v i s}\right) I_{n}\left(\omega_{I R}\right)$

The indices $l m n$ stand for light-polarization directions in the order sum-frequency, visible and infrared. Experiments are typically carried out with the incident beams propagated in a single plane that is perpendicular to the probing surface. The possible polarizations are then $\mathrm{S}$ or $\mathrm{P}$, corresponding to polarization perpendicular or parallel to the plane of incidence, respectively.

The effective susceptibility is related to the true second-order susceptibility $\chi_{i j k}$ by Fresnel factors and unit polarization vectors for each beam. The susceptibility can be represented as the sum of resonant and non-resonant components. The later is almost independent of the frequency, while the former contains the important information on vibrational states: the SFG signal is enhanced when $\omega_{I R}$ approaches the frequency $\omega_{Q}$ of a vibrational mode. The second-order susceptibility tensor $\chi_{i j k}$ has 27 elements but for symmetry reasons, only certain elements are non-zero and unique. ${ }^{15-16}$ Moreover, if the liquid surface is isotropic, azimuthal symmetry makes only 7 elements to be non-zero: $\chi_{x x z}=\chi_{y y z}, \chi_{x z x}=\chi_{y z y}, \chi_{z x x}=\chi_{z y y}$, and $\chi_{z z z}$. If in addition $\omega_{v i s}$ is far from resonance, only three terms are non-zero and independent, as $\chi_{x z x}=\chi_{y z y}=$ $\chi_{z x x}=\chi_{z y y} \cdot{ }^{16-17}$ These elements can be determined using different combinations of the polarization conditions. For instance, in the widely used SSP polarization scheme, if we assume 
$Z$ to be the surface normal and the incident light being along the $X Z$ plane, the susceptibility element relevant to the SFG signal is simply $\chi_{y y z}$ (or equivalently $\chi_{x x z}$ ).

Different approaches are available to calculate the resonant component of the macroscopic susceptibility at liquid, ${ }^{18-22}$ or solid interfaces, ${ }^{23-28}$ but most of the time it is computed in the timerepresentation, i. e. using the classical time-correlation function (TCF) of the polarizability and dipole moment of the system from a Molecular Dynamics (MD) simulation: ${ }^{29}$

$c_{p q r}(t)=\left\langle\alpha_{p q}(t) \mu_{r}(0)\right\rangle$

where $\alpha(t)$ and $\mu(0)$ are, respectively, the polarizability at time $t$ and the dipole moment at time 0 , the indices $p-q-r$ are Cartesian coordinates in a space-fixed axis system, and the brackets mean an average over the simulation. This TCF represents the classical version of the quantum mechanical TCF, to which it is related by simple approximated expressions. ${ }^{22}$ The resonant second-order susceptibility tensor is then obtained by the Fourier-Laplace transform:

$\chi_{p q r}(\omega)=\frac{i \omega}{k_{B} T} \int_{0}^{\infty} e^{i \omega t} c_{p q r}(t) d t$

where $k_{B}$ is the Boltzmann constant and T the temperature. An approximated form of (3) has also been proposed by assuming a linear variation of the dipole moment and polarizability with system coordinates, so that $c(t)$ takes the form of a velocity-velocity time-correlation function. ${ }^{30-}$ 32

Most calculations have been carried out using classical MD simulations and parametric expressions for the molecular dipole moment and polarizability. ${ }^{19,29}$ In a number of cases, ${ }^{29,33-35}$ the classical MD trajectory provides the input to combined QM/MM computations of the molecular dipole moment and polarizability, in which part of the system (typically the solute molecule) is treated Quantum Mechanically (QM) while the surrounding system (the solvent) is 
treated with a Molecular Mechanics (MM) force-field. More rigorous SFG calculations would require the use of first-principles MD simulations, but in practice, due to high computational cost and slow convergence of the time correlation function, only few simulations of this type have been reported so far, using either full ab initio ${ }^{36-38}$ or QM/MM schemes. ${ }^{39}$

In this work, we propose a cost-effective method for highly-accurate first-principles calculations of the SFG signal that complements the list of available tools. The method is based, on one hand, on expressing the second-order susceptibility tensor in the energy representation scheme, i. e. as a configurational average rather than a time-correlation function, which is straightforward to do if one assumes the harmonic approximation. Anharmonicities can be estimated in subsequent steps. On the other hand, it makes use of the QM/MM dual-level methodology developed before to calculate accurate configurational averages in the framework of perturbation theory..$^{40-42}$

We apply the method to methanol at the air-water interface because this is one of the simplest amphiphilic surfactant model systems, ${ }^{43-53}$ and in addition, the SFG spectrum in the $\mathrm{CH}_{3}$ stretching domain has been thoroughly studied both experimentally and theoretically, using classical MD simulations, in different conditions. ${ }^{54-63}$ It is therefore a convenient case study to test the proposed methodology. We also briefly consider the case of acetonitrile as an example to show the suitability of the method to predicting subtle modifications of the $\mathrm{CH}_{3}$-signals in different chemical surroundings.

\section{Theoretical model}

SFG signal in the energy representation. To get $\chi(\omega)$ as a configurational average, let us first rewrite the Fourier-Laplace transform (3) in terms of its Fourier transform, $C(w)$, taking 
advantage of the fact that $c(t)$ is a classical time correlation function and therefore it is a real and even function. ${ }^{64}$ Since $c(t)$ is real and even, so is $C(w)$ (though only positive frequencies are relevant to our purpose here). Now, changing the integration limits of $(3)$ to $(-\infty,+\infty)$ after multiplying $c(t)$ by the Heaviside function $u(t)(u(t)=0$ for $t<0, u(t)=1$ for $t \geq 0)$, one gets:

$\chi(\omega)=i \frac{\pi \omega}{k_{B} T} C(\omega)-\frac{\omega}{k_{B} T} \int_{-\infty}^{+\infty} \frac{C(\omega-\omega \prime)}{\omega \prime} d \omega^{\prime}$

To derive (4), we use the convolution theorem and the Fourier transform of the Heaviside function, $U(\omega)=\frac{1}{i \omega}+\pi \delta(\omega)$. Equation (4) highlights the important fact that the real and imaginary parts of the susceptibility (the Fourier-Laplace transform of a causal function) are not independent but interrelated by a Hilbert transform.

If we now assume the harmonic approximation, $\chi(\omega)$ can be calculated to first order in terms of the dipole moment and polarizability derivatives with respect to the normal modes. As shown by Morita and Hynes, ${ }^{18}$ the contribution of mode $Q$ of frequency $\omega_{I R}$ to $\chi(\omega)$ is then:

$\chi_{p q r}^{Q}(\omega) \propto\left(\frac{\partial \alpha_{p q}}{\partial Q}\right)\left(\frac{\partial \mu_{r}}{\partial Q}\right)\left[\frac{\omega-\omega_{I R}}{\left(\omega-\omega_{I R}\right)^{2}+\gamma^{2}}+\frac{i \gamma}{\left(\omega-\omega_{I R}\right)^{2}+\gamma^{2}}\right]$

where a Lorentzian shape (whose real and imaginary parts are related by a Hilbert transform) is assumed for the SFG signal and $\gamma$ is a damping parameter. Expression (5) opens the way to calculating the SFG spectrum as a configurational average from MD simulations using timedependent frequencies and "instantaneous normal modes" (INM). The INM approach ${ }^{65-67}$ has been widely used in infrared spectroscopy, despite some well-known drawbacks. ${ }^{68}$ Vibrational spectra are obtained as a weighted density of states from instantaneous spectroscopic properties calculated using a set of MD snapshots. Very few applications of the INM approach have been reported in SFG spectroscopy, but Perry et $\mathrm{al}^{22,69}$ reported expressions to calculate the susceptibility from the associated $C_{p q r}^{Q}(\omega)$ function: 
$C_{p q r}^{Q}(\omega)=k_{B} T\left\langle\left(\frac{\partial \alpha_{p q}}{\partial Q}\right)\left(\frac{\partial \mu_{r}}{\partial Q}\right) \frac{\delta\left(\omega-\omega_{I R}\right)}{\omega^{2}}\right\rangle$

where $\delta$ is effectively transformed into a bin size, ${ }^{22}$ and the average is calculated using a set of solute-solvent configurations from the simulations. The SFG signal is finally obtained by replacing (6) into (4). Alternatively, (6) may be back transformed into the time domain to yield $c^{H}(t)$ and the SFG signal computed through equation (3). Following the proposal by Morita and Hynes, ${ }^{18}$ a Lorentzian-shaped band like (5) can be assumed in the calculation of $\chi(\omega)$ for each instantaneous normal mode, so that the weighted density of states is obtained as an average of individual Lorentzian signals.

The INM-FEM method. INM calculations provide complementary information to TCF data because the SFG signal can be decomposed in terms of normal modes contributions, which is extremely useful for the interpretation of experimental spectra. However, SFG resonances have been found to be significantly broader in INM calculations, strongly limiting its usefulness. ${ }^{22,70}$ This finding has been interpreted as a limitation of the method to capture solvent dynamical effects such as motional narrowing or vibrational dephasing, which are naturally incorporated into the TCF method..$^{71}$

In the context of the QM/MM Molecular dynamics simulations used here, the shortcomings of the INM approach in infrared spectroscopy have been discussed in detail before. ${ }^{72-73}$ In these works, the authors proposed a time-resolved method coupled to a generalized-virial frequency calculation that can account for motional narrowing. ${ }^{73}$ The method led to inhomogeneous band broadening in very good agreement with the Fourier transforms of the time correlation functions. Moreover, it was shown that similar bandwidths can be obtained with a variant of the standard 
INM technique (the so-called INMA or INM-analysis technique $)^{74}$ in which the energy of the solute is minimized in the solvent cage prior to computing the instantaneous Hessian and associated frequencies. This result was interpreted as a means of escaping situations with strong collision interactions by reducing the fluctuations that are responsible for the overestimation of the INM bandwidths. ${ }^{73}$ The INMA approach involves therefore energy minimization of the solute structure for each configuration in the sampling. Though this method is computationally more efficient that the full solute-solvent minimization proposed by other authors,${ }^{75}$ it faces some fundamental and practical problems. On one hand, the physical meaning of the partially relaxed structures is not clear, ${ }^{74}$ and therefore the interpretation of the obtained averages may be problematic. On the other hand, even though the solute's atomic coordinates are only relaxed, the computational effort is significant because the convergence of the minimization procedure turns to be slow often in such complex environments. This point is particularly relevant when one considers the potential application of the method to the case of interfaces. Indeed, at interfaces, the minimization of the solute's coordinates can lead to configurations extremely far from the initial configuration when the solute is not tightly bonded to the interface and tend to "glide" over the surface.

Recently, another variant of the INM and INMA approaches has been reported to study the vibrational spectra of a solute in a bulk solvent. ${ }^{76}$ In this INM technique,${ }^{76}$ the solute-solvent vibrational motions along the time-dependent trajectory are decoupled and the INM are obtained from the configurational ensemble that expands a free energy minimum (FEM) of the system with respect to variations in the solute's atomic coordinates. To locate the FEM, an iterative procedure based on the free-energy gradient (FEG) theory is used, searching for a solute's internal geometry at which: ${ }^{.77}$ 
$\frac{\partial \mathrm{G}(\boldsymbol{q})}{\partial \boldsymbol{q}}=\left\langle\frac{\partial V(\boldsymbol{q})}{\partial \boldsymbol{q}}\right\rangle \simeq 0$

where $\boldsymbol{q}$ are nuclear mass-weighted atomic Cartesian coordinates of the solute and $V$ is the potential energy of the solute-solvent system. In addition, the free-energy Hessian with respect to $\boldsymbol{q}$ coordinates $\boldsymbol{H}^{\mathrm{FE}}(\boldsymbol{q})$ verifies:

$\boldsymbol{H}^{\mathrm{FE}}(\boldsymbol{q}) \simeq\left\langle\frac{\partial^{2} V(\boldsymbol{q})}{\partial \boldsymbol{q} \partial \boldsymbol{q}}\right\rangle$

The instantaneous Hessian matrices $\partial^{2} V(\boldsymbol{q}) / \partial \boldsymbol{q} \partial \boldsymbol{q}$ are then diagonalized to obtain the instantaneous normal modes and the corresponding time dependent frequencies for a set of configurations. ${ }^{76}$ The vibrational spectrum is obtained in a way similar to the standard INM approach through a weighted density of states. The INM-FEM and previous INM and INMA methods, therefore, differ essentially by the way the configurational sampling describing the instantaneous normal modes are defined.

One should note that in this INM-FEM approach, the focus is put on the solute's vibrational frequencies, assuming that they can be decoupled from the solvent vibrations. This is consistent with the use of multiscale QM/MM methods but in some cases, e.g. when the target solute's vibrations appear in the same frequency regions than the solvent, the Hessian matrix to be diagonalized must account for potential couplings. In such instances, one has to consider moving to a full QM treatment (ab initio molecular dynamics techniques) or possibly to an adaptative QM/MM approach in which some solvent molecules are explicitly included in the QM part. The treatment proposed here can still be implemented but of course the computational cost becomes much higher. 
SFG signal in the combined FEP-INM-FEM approach. To date, no application of the INMFEM method to SFG vibrational spectroscopy of interfaces has been carried out. The approach that we propose here explores such possibility, but in order to attain high ab initio accuracy, the INM-FEM method is combined with free-energy perturbation (FEP) theory. Standard FEP theory ${ }^{81}$ provides a means to obtain the free energy change of a system accompanying a perturbation such as the interaction with an external electric field or a solvent, for instance. One gets:

$\Delta G=G_{1}-G_{0}=-\frac{1}{\beta} \ln \left\langle e^{-\beta \Delta V}\right\rangle_{0}$

where $\Delta V=V_{1}-V_{0}$ is the potential energy change for a given configuration, and the average is taken over equilibrium configurations of the non-perturbed system. Likewise, for any property $A$ depending on the system coordinates, one deduces:

$A_{1}=\frac{\left\langle\mathrm{e}^{-\beta \Delta V} A_{1}\right\rangle_{0}}{\left\langle\mathrm{e}^{-\beta \Delta V)}\right\rangle_{0}}$

In our FEP approach, the perturbation does not correspond to a change of the physical conditions of the system, but rather to a change in the level of elaboration of the system Hamiltonian (forcefield) describing it. For example, the perturbation can be the upgrading of the quantum mechanical level in QM/MM simulations, or more generally, the transition from a low-level to a high-level Hamiltonian ( $H_{L L}$ and $H_{H L}$, respectively), which can be any appropriate combination of quantum and/or classical, force-fields. Such a dual-level model has previously been developed for the calculation of accurate free-energy $y^{40,53,82}$ and free energy derivatives ${ }^{41-42}$ from ab initio or QM/MM MD simulations. The convergence of this dual-level scheme depends on several factors that have been discussed in detail elsewhere. ${ }^{40-42,53,82}$ 
Application of the FEP theory to the INM-FEM method encompasses two steps. First, one has to minimize the free energy of the system at the high-level, for which the following free-energy gradient can be used: ${ }^{41}$

$$
\frac{\partial G_{H L}(\boldsymbol{q})}{\partial \boldsymbol{q}}=\left\langle\frac{\partial V_{H L}(\boldsymbol{q})}{\partial \boldsymbol{q}}\right\rangle_{\mathrm{HL}} \simeq \frac{1}{\left\langle\mathrm{e}^{-\beta \Delta V(\boldsymbol{q})}\right\rangle_{\mathrm{LL}}}\left\langle\mathrm{e}^{-\beta \Delta V(\boldsymbol{q})} \frac{\partial V_{H L}(\boldsymbol{q})}{\partial \boldsymbol{q}}\right\rangle_{\mathrm{LL}}
$$

where $\Delta V=V_{H L}-V_{L L}$ and the other symbols an obvious meaning. After free energy minimization, the approximate value of any property such as $\chi(\omega)$ at high-level is given by: ${ }^{81}$

$\chi(\omega) \simeq \frac{\left\langle\mathrm{e}^{-\beta \Delta V} \chi_{H L}(\omega)\right\rangle_{\mathrm{LL}}}{\left\langle\mathrm{e}^{-\beta \Delta V)}\right\rangle_{\mathrm{LL}}}$

where the explicit dependence on the solute's normal modes and geometry has been omitted for simplicity.

\section{Computational details}

We focus on the SSP SFG signal in the $\mathrm{CH}_{3}$ stretching region of methanol at the air-water interface, whose assignment has caused some debate in the literature. The PPP spectrum has also been calculated and is provided as SI. For comparison, a calculation of the SSP band in the case of acetonitrile is presented too.

The target theoretical level here $\left(H_{H L}\right)$ is a QM/MM scheme in which the solute is described quantum mechanically at the B3LYP level ${ }^{83}$ using the $6-311+\mathrm{G}(\mathrm{d})$ basis set $^{84-86}$ and water molecules are described using a flexible TIP3P force-field ${ }^{87-88}$ Though much higher ab initio levels, such as CCSD, could be considered in our FEP-INM-FEM approach, the choice of the B3LYP/6-311+G(d) method corresponds to the following criteria. First, in this study, the goal is to test the accuracy of the developed method, and to this aim, MD simulations at the same QM 
level and rigorous SFG calculations using the TCF approach must be carried out, which would be impossible at MP2, CCSD or other ab initio correlated levels. Second, preliminary tests (Table S1) showed that B3LYP/6-311+G(d) provides an excellent approximation to harmonic frequencies, and in addition, comparison of B3LYP and CCSD calculations for SFG intensities showed a qualitatively good agreement. ${ }^{89}$ For the low-level $\left(H_{L L}\right)$ we simply use here an adapted OPLS classical force-field (see SI). ${ }^{90}$ MD simulations assume the NVT ensemble ( $\mathrm{T}=298 \mathrm{~K}$, Nosé-Hoover thermostat ${ }^{91-92}$ ) and a box containing the solute and 499 water molecules ( $24.685 \mathrm{x}$ 24.685 x $130 \AA$ ) with periodic boundary conditions along XY directions. The time step is $0.25 \mathrm{fs}$ in QM/MM simulations and 0.5 fs in classical force-field simulations. For the SFG computation, after free-energy minimization with respect to the solute's coordinates, 1000 snapshots were selected from a $100 \mathrm{ps}$ low-level simulation, and for each snapshot $\chi(\omega)$ was calculated at the high-level. The average was obtained after applying the appropriate configuration weighting, like in equation 6. To test the accuracy of the FEP-INM-FEM approach, we also carried out a MD simulation at the high-level, and calculated the spectrum in the time representation using the TCF methodology. The QM/MM simulation was carried out for 300 picosenconds and the appropriate TCF calculated up to 8 ps. We use Gaussian $09^{93}$ for QM calculations, Tinker $4.2^{94}$ for classical simulations and the interface developed by us to carry out the combined QM/MM simulations. ${ }^{95}$ Other details are provided as Supporting Information.

\section{Results and discussion}

The experimental SSP SFG signal of the methanol molecule at the air-water interface displays two distinct peaks in the $\mathrm{CH}_{3}$ stretching domain. ${ }^{54-63}$ The most intense peak appears at around $2840 \mathrm{~cm}^{-1}$ and is associated to the symmetric stretch of the $\mathrm{CH}_{3}$ group, while the second, less 
intense peak appears at higher energy, in the $2920-2945 \mathrm{~cm}^{-1}$ range, i. e., in the frequency domain of the asymmetric $\mathrm{CH}_{3}$ stretch ${ }^{96}$ (the $\mathrm{CH}_{3}$ stretching frequencies of methanol in the gasphase $\operatorname{are}^{97} 2844 \mathrm{~cm}^{-1}$ (symmetric), and 2970 and $2999 \mathrm{~cm}^{-1}$ (asymmetric)). However, the less intense peak in the SFG spectrum has been associated to Fermi resonances..$^{54,58,98-100}$

The accuracy of the calculated SSP components of the susceptibility tensor using the FEP-INMFEM approach were first tested by comparing the results with the rigorous TCF calculation from the QM/MM simulation. The comparison is made in Figure 1. One can notice that the TCF-based susceptibility curves contain considerable noise that could not be further reduced using standard signal treatment filters. The noise is principally due to a limited simulation time (300 ps) and the slow convergence of the TCF. Unfortunately, at the QM/MM level considered here and due to the high computational cost, it was not possible to carry out longer simulations. Indeed, the QM/MM simulation involves one B3LYP/6-311+G(d) calculation at each time step to calculate the energy, the forces, the dipole moment and the polarizabilities. Thus, the $300 \mathrm{ps}$ trajectory required $1.210^{6}$ calculations of this type representing about 70 days of CPU time (Intel Xeon E52630 v3 processor, $2.4 \mathrm{GHz}$ ). However, the comparison in Figure 1 shows that the general shape of the imaginary and real parts of the reference TCF signal is very well reproduced by the FEPINM-FEM method, demonstrating the reliability of the approach for the calculation of SFG spectra at interfaces. 


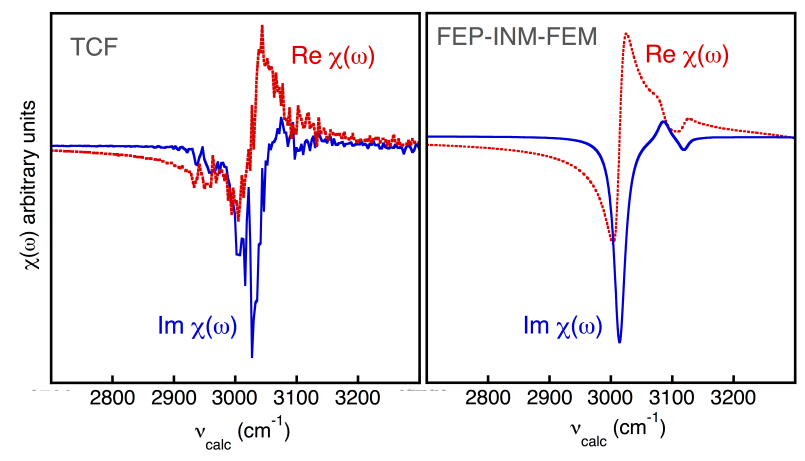

Figure 1. Calculated SSP component of the susceptibility tensor of methanol at the air-water interface. Left: rigorous calculation using the TCF from QM/MM MD simulations. Right: calculation using our approximate FEP-INM-FEM method.

The comparison with experiment in the $\mathrm{CH}_{3}$-stretching region is done in Figure 2 (the full SSP spectrum of methanol calculated in this work is shown in Figure S1). It is worth doing two preliminary remarks. First, the experimental curve in Figure 2 corresponds in fact to the Lorentzian functions that fit the experimental data, as reported by Ma and Allen ${ }^{58}$ for low molefraction aqueous methanol solutions. Second, since the FEP-INM-FEM calculations are based on $\mathrm{ab}$ initio harmonic frequencies, a frequency shift is necessary before comparison with experiment is done. In the Figure, we have shifted the $\mathrm{X}$-axes so that the first peak in the experimental spectrum, which corresponds to the symmetric $\mathrm{CH}_{3}$ stretching frequency, coincides with our calculated peak; the shift amounts $174 \mathrm{~cm}^{-1}$. Since the calculated harmonic frequencies at B3LYP/6-311+G(d) level are very close to calculations at much higher levels of theory (Table $\mathrm{S} 1)$, numerical errors inherent to the ab initio level used (density functional, basis set) are not expected to play a significant role in the experimental-theoretical frequency differences. The later must indeed be ascribed to anharmonic effects. Actually, the shift is very close to the 
B3LYP/6-311+G(d) anharmonic correction calculated in gas phase for the symmetric $\mathrm{CH}_{3}$ stretching mode: the harmonic and anharmonic frequencies are, respectively, $2999 \mathrm{~cm}^{-1}$ and 2818 $\mathrm{cm}^{-1}$ (Table S1), leading to a correction of $181 \mathrm{~cm}^{-1}$.

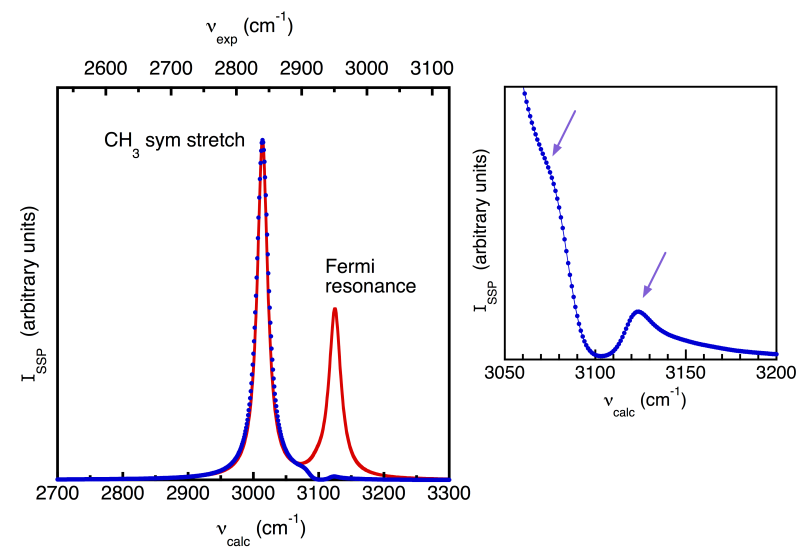

Figure 2. Calculated SSP SFG spectrum of methanol at the air-water interface using our approximate FEP-INM-FEM method. In the left panel, we compare the calculated spectrum (blue circles) with the experimental curve (plain red line) after an appropriate frequency shift to account for anharmonicites (see text). The inset in the right panel zooms over the calculated spectrum in the asymmetric $\mathrm{CH}_{3}$ stretching region. The experimental curve corresponds to the Lorentzian fit reported before for low mole-fraction aqueous methanol solutions. ${ }^{58}$

As shown in Figure 2, our calculations predict a single strong and sharp band in the $\mathrm{CH}_{3}-$ stretching region $\left(\mathrm{FWHM} \sim 40 \mathrm{~cm}^{-1}\right.$ ), which extends within roughly $\pm 100 \mathrm{~cm}^{-1}$ and fits almost perfectly the corresponding experimental curve. This is a main result of this work, which supports the fact that introducing dynamics effects in SFG spectra computations through solvent fluctuations around the free-energy minimum, as done in the INM-FEM method, represents a 
significant improvement over standard INM approaches with QM/MM MD simulations, whose deficiencies are well-known. ${ }^{72-74}$ To illustrate the limitations of the later approach, Figure S2 displays the vibrational density of states obtained when the standard INM method is used, i. e. when the frequencies are calculated using non-relaxed snapshots from the QM/MM simulation carried out with a flexible solute geometry. It is clearly shown that the bandwidth in the $\mathrm{CH}_{3}$ region is about one order of magnitude larger than the corresponding experimental SFG band, or the bands calculated with either the FEP-INM-FEM method (Figure 2) or the TCF approach (not shown). It overlaps the VDOS band corresponding to the methanol OH stretching vibration. Hence, one cannot expect to get reliable SFG spectra using this approach without a specific dynamic treatment similar for example to that used for QM/MM computations of infrared spectra in bulk solution. ${ }^{73}$

As mentioned, the peak at higher energy in the experimental SSP SFG spectrum of methanol at the air-water interface has been ascribed to a Fermi resonance that cannot be reproduced with our theoretical scheme. Nevertheless, this peak appears in the same region as the asymmetric stretching vibrations, which have caused some discussion in the literature to clarify the possible contribution of these vibrational modes. Interestingly, a zoom over the upper energy tail of the calculated band shown in the inset of Figure 2, reveals the presence of two small additional features (marked by arrows), arguably corresponding to the contribution of the asymmetric stretching vibrations. To further analyze this point, a separate calculation of the symmetric and asymmetric mode contributions to the SFG spectrum has been carried out by neglecting the symmetric-asymmetric cross-terms in the calculation of $|\chi(\omega)|^{2}$, and the result is plotted in Figure 3. The $\mathrm{CH}_{3}$ asymmetric band is found to be around 100 times smaller than the symmetric one, and thus its contribution can be neglected in the case of methanol. 


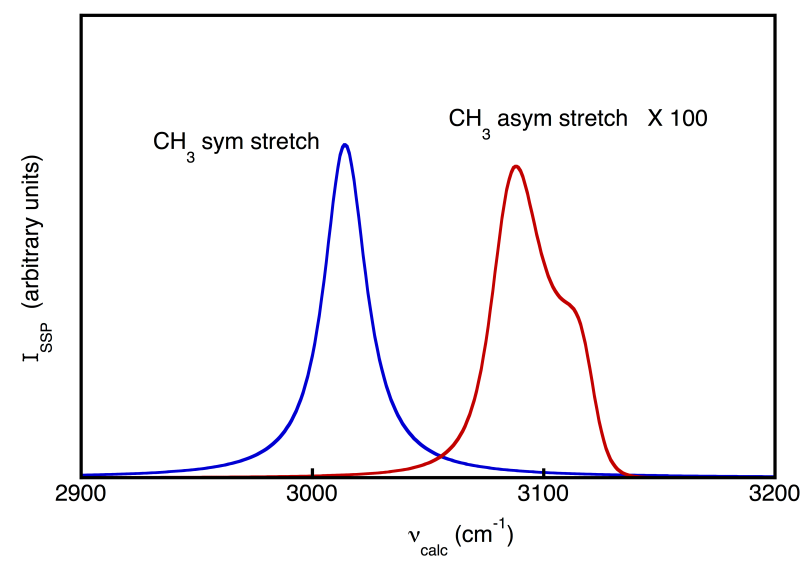

Figure 3. Separated $\mathrm{CH}_{3}$ symmetric and asymmetric stretching mode contributions to the SFG spectrum of methanol at the air-water interface using the FEP-INM-FEM approach (cross-terms are not included). The asymmetric modes band intensity has been multiplied by100 to facilitate the comparison.

However, SFG spectra are by nature quite sensitive to orientational effects and it is interesting to see how the symmetric and asymmetric $\mathrm{CH}_{3}$ stretching vibrations change with orientation. This issue has been theoretically analyzed for a static alkyl chain model at CCSD level of theory ${ }^{89} \mathrm{In}$ the case of methanol (Figure S3), the $\mathrm{COH}$ group is most of the time oriented towards the water layer, with the $\mathrm{CO}$ bond being almost parallel to the Z-axis perpendicular to the water surface $\left(\theta=180^{\circ}\right)$. However, the simulation shows that a significant fraction of configurations depart from this preferred orientation. Figure 4 shows that for angles close to $120^{\circ}$ or smaller, the asymmetric modes (specially the lower asymmetric mode) give rise to a band with significant intensity compared to the symmetric mode, that still prevails. The case $\theta=90^{\circ}$ is a particular one because due to its low configuration fraction (about 1\%), results are probably not statistically meaningful. Nevertheless, it shows that when the $\mathrm{CH}_{3}$-group axis is parallel to the water surface, the asymmetric contribution can be larger than the symmetric one. One must also note that the 
whole SFG intensity strongly decreases with the value of $\theta$; for instance, the ratio between the integrated areas of the $\mathrm{CH}_{3}$ stretching bands for $\theta=170^{\circ}$ and $\theta=100^{\circ}$ is 20 . These findings are consistent with those reported before considering a static gas-phase model of an alkyl chain, ${ }^{89}$ and provide insights that may be helpful for the analysis of the SFG spectrum of more complex molecules bearing methyl groups at the air-water interface.

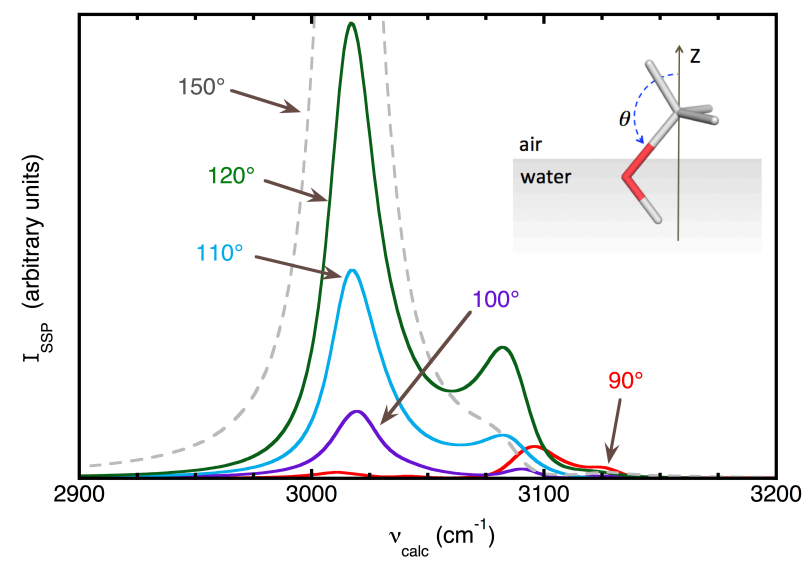

Figure 4. SSP SFG spectra of methanol at the air-water interface as a function of methanol orientation using the approximate FEP-INM-FEM method. Each curve represents the average spectrum for configurations displaying a given angle $\left( \pm 5^{\circ}\right)$. The fraction of configurations decreases with the value of the angle, as shown in Figure $\mathrm{S} 2$ for $\mathrm{P}(\cos (\theta))$. For angles greater than $150^{\circ}$, the shapes of the spectra are quite similar (and similar to the total spectrum in Figure 2), though the absolute intensities increase progressively. For simplicity, they are not reproduced in the Figure.

Before concluding this discussion, it is worth emphasizing that any theoretical tool intended to help interpreting experimental SFG spectra must be able to reproduce the changes observed in different polarization setups, as well as the small spectral shifts produced by chemical changes 
such as group substitutions. To check these specific issues, two additional tests of our method have been performed. First, we have made a comparison of the SSP and PPP signals in the case of methanol. This comparison requires to account for Fresnel factors and incident beam angles, which makes the analysis somewhat more complicated. The results shown in Figure S4 have been obtained using the approximations made by Carr et al, ${ }^{101}$ and appear to be in qualitative good agreement with previous calculations and experimental data. ${ }^{61,63}$ In particular, the sign of the ratio between the imaginary parts of the PPP and SSP signals is negative at the symmetric $\mathrm{CH}_{3}$-stretch absorption frequency, and amounts -0.2 , which has the same order of magnitude that the ratio estimated with other methods. ${ }^{61}$ Moreover, we predict a quite small intensity of the SFG band in the PPP polarization (relative to the SSP signal) that agrees with reported SFG spectra using different experimental setups. ${ }^{63}$

In the second test, we have compared the methanol and acetonitrile SSP bands for the symmetric $\mathrm{CH}_{3}$-stretch. The calculations are shown in Figure S5. The experimental signal for acetonitrile ${ }^{102-}$

${ }^{103}$ displays a maximum around $2944 \mathrm{~cm}^{-1}$, which represents a blue shift with respect to methanol of $\sim 100 \mathrm{~cm}^{-1}$. The FEP-INM-FEM results are satisfactory in this case too. They correctly predict the subtle shift produced by the change in the methyl-bonded chemical group (a cyano group instead of an alcohol group), although the predicted absolute value is slightly lower than that observed, i.e. $24 \mathrm{~cm}^{-1}$, or $32 \mathrm{~cm}^{-1}$ when anharmonic corrections are taken into account.

\section{Conclusions}

In summary, we have developed a new FEP-INM-FEM approach to compute SFG spectra that complements the list of currently available computational tools and opens up interesting perspectives for the interpretation of experimental data. First, FEP-INM-FEM calculations 
provide very useful information that is not provided by TCF data, because the SFG signal is decomposed in terms of normal mode contributions, which is a key advantage for assignment of the observed bands. Second, the accuracy of the approach is comparable to that of rigorous computations using time-correlation functions and ab initio simulations but its computational cost is much lower. The CPU cost reduction depends on the choice of the high- and low-level Hamiltonians but as in other FEP applications ${ }^{41-42}$ it may represent up to 2-3 orders of magnitude in comparison to standard calculations. Moreover, the wall-clock time is also drastically reduced because calculations for independent configurations can benefit from parallel computing, while the TCF QM/MM simulations remain essentially serial, using a limited number of processors. Therefore, though a simple system has been described here to test the suitability of the method by comparing with rigorous TCF calculations, the FEP-INM-FEM approach is designed to deal with much larger systems or with the most elaborated quantum chemical methods.

\section{ASSOCIATED CONTENT}

Supporting Information. The following files are available free of charge.

Computational details. Table S1 (calculated and experimental frequencies of methanol). Figures S1-S5. (PDF)

\section{AUTHOR INFORMATION}

\section{Notes}

The authors declare no competing financial interests. 


\section{ACKNOWLEDGMENT}

The authors acknowledge the French CINES for providing computational resources (Project LCT2550). They also thank Dr. Francesca Ingrosso (University of Lorraine, France) and Prof. Adolfo Bastifa (University of Murcia, Spain) for fruitful discussions about the INM theory.

\section{REFERENCES}

1. Shen, Y. R. Surface-properties probed by $2^{\text {nd }}$-harmonic and sum-frequency generation. Nature 1989, 337, 519-525.

2. Eisenthal, K. Liquid interfaces probed by second-harmonic and sum-frequency spectroscopy. Chem. Rev. 1996, 96, 1343-1360.

3. Shen, Y. Fundamentals of sum-frequency spectroscopy. Cambridge University Press: Cambridge, 2016.

4. Tang, F.; Ohto, T.; Sun, S.; Rouxel, J. r. m. R.; Imoto, S.; Backus, E. H.; Mukamel, S.;

Bonn, M.; Nagata, Y. Molecular Structure and Modeling of Water-Air and Ice-Air Interfaces Monitored by Sum-Frequency Generation. Chem. Rev. 2020, 120, 3633-3667.

5. McGuire, J. A.; Shen, Y. R. Ultrafast vibrational dynamics at water interfaces. Science 2006, 313, 1945-1948.

6. Bonn, M.; Bakker, H. J.; Ghosh, A.; Yamamoto, S.; Sovago, M.; Campen, R. K. Structural Inhomogeneity of Interfacial Water at Lipid Monolayers Revealed by Surface-Specific Vibrational Pump-Probe Spectroscopy. J. Am. Chem. Soc. 2010, 132, 14971-14978. 
7. Eftekhari-Bafrooei, A.; Borguet, E. Effect of Hydrogen-Bond Strength on the Vibrational Relaxation of Interfacial Water. J. Am. Chem. Soc. 2010, 132, 3756-3761.

8. Zhang, Z.; Piatkowski, L.; Bakker, H. J.; Bonn, M. Communication: Interfacial water structure revealed by ultrafast two-dimensional surface vibrational spectroscopy. J. Chem. Phys. 2011, 135, Art. No. 021101.

9. Zhang, Z.; Piatkowski, L.; Bakker, H. J.; Bonn, M. Ultrafast vibrational energy transfer at the water/air interface revealed by two-dimensional surface vibrational spectroscopy. Nature Chem. 2011, 3, 888-893.

10. Xiong, W.; Laaser, J. E.; Mehlenbacher, R. D.; Zanni, M. T. Adding a dimension to the infrared spectra of interfaces using heterodyne detected 2D sum-frequency generation (HD 2D SFG) spectroscopy. Proc. Natl. Acad. Sci. USA 2011, 108, 20902-20907.

11. Inoue, K.-i.; Nihonyanagi, S.; Singh, P. C.; Yamaguchi, S.; Tahara, T. 2D heterodynedetected sum frequency generation study on the ultrafast vibrational dynamics of $\mathrm{H} 2 \mathrm{O}$ and HOD water at charged interfaces. J. Chem. Phys. 2015, 142, Art. No. 212431.

12. Backus, E. H. G.; Cyran, J. D.; Grechko, M.; Nagata, Y.; Bonn, M. Time-Resolved Sum Frequency Generation Spectroscopy: A Quantitative Comparison Between Intensity and PhaseResolved Spectroscopy. J. Phys. Chem. A 2018, 122, 2401-2410.

13. Shen, Y. Surface spectroscopy by nonlinear optics. In Proc. Int. School of Physics Enrico Fermi, Holland, N., Ed. Verenna, Italy: International School of Physics-Enrico Fermi: Amsterdam, 1994; Vol. 139, p 139. 
14. Miranda, P.; Shen, Y. Liquid interfaces: A study by sum-frequency vibrational spectroscopy. J. Phys. Chem. B 1999, 103, 3292-3307.

15. Lambert, A. G.; Davies, P. B.; Neivandt, D. J. Implementing the Theory of Sum Frequency Generation Vibrational Spectroscopy: A Tutorial Review. Applied Spectroscopy Reviews 2005, 40, 103-145.

16. Richmond, G. Molecular bonding and interactions at aqueous surfaces as probed by vibrational sum frequency spectroscopy. Chem. Rev. 2002, 102, 2693-2724.

17. Kido Soule, M. C.; Hore, D. K.; Jaramillo-Fellin, D. M.; Richmond, G. L. Differing Adsorption Behavior of Environmentally Important Cyanophenol Isomers at the Air- Water Interface. J. Phys. Chem. B 2006, 110, 16575-16583.

18. Morita, A.; Hynes, J. T. A theoretical analysis of the sum frequency generation spectrum of the water surface. Chem. Phys. 2000, 258, 371-390.

19. Morita, A.; Hynes, J. T. A theoretical analysis of the sum frequency generation spectrum of the water surface. II. Time-dependent approach. J. Phys. Chem. B 2002, 106, 673-685.

20. Morita, A.; Ishiyama, T. Recent progress in theoretical analysis of vibrational sum frequency generation spectroscopy. Phys. Chem. Chem. Phys. 2008, 10, 5801-5816.

21. Morita, A. Improved computation of sum frequency generation spectrum of the surface of water. J. Phys. Chem. B 2006, 110, 3158-3163. 
22. Perry, A.; Neipert, C.; Space, B.; Moore, P. B. Theoretical modeling of interface specific vibrational spectroscopy: Methods and applications to aqueous interfaces. Chem. Rev. 2006, 106, 1234-1258.

23. Zheng, D. S.; Wang, Y.; Liu, A. A.; Wang, H. F. Microscopic molecular optics theory of surface second harmonic generation and sum-frequency generation spectroscopy based on the discrete dipole lattice model. Int. Rev. Phys. Chem. 2008, 27, 629-664.

24. Cecchet, F.; Lis, D.; Guthmuller, J.; Champagne, B.; Fonder, G.; Mekhalif, Z.; Caudano, Y.; Mani, A. A.; Thiry, P. A.; Peremans, A. Theoretical calculations and experimental measurements of the vibrational response of p-NTP SAMs: An orientational analysis. J. Phys. Chem. C. 2010, 114, 4106-4113.

25. Feugmo, C. G. T.; Liegeois, V.; Champagne, B. Theoretical Investigation of Vibrational Sum-Frequency Generation Signatures of Functionalized H-Si(111). J. Phys. Chem. C 2015, $119,3180-3191$.

26. Wan, Q.; Galli, G. First-Principles Framework to Compute Sum-Frequency Generation Vibrational Spectra of Semiconductors and Insulators. Phys. Rev. Lett. 2015, 115, Art. No. 246404.

27. Chase, H. M.; Chen, S.; Fu, L.; Upshur, M. A.; Rudshteyn, B.; Thomson, R. J.; Wang, H.-F.; Batista, V. S.; Geiger, F. M. Orientations of nonlocal vibrational modes from combined experimental and theoretical sum frequency spectroscopy. Chem. Phys. Lett. 2017, 683, 199-204.

28. Pezzotti, S.; Galimberti, D. R.; Shen, Y. R.; Gaigeot, M.-P. Structural definition of the BIL and DL: A new universal methodology to rationalize non-linear $\chi^{(2)}(\omega)$ SFG signals at 
charged interfaces, including $\chi^{(3)}(\omega)$ contributions. Phys. Chem. Chem. Phys. 2018, 20, 51905199.

29. Ishiyama, T.; Imamura, T.; Morita, A. Theoretical Studies of Structures and Vibrational Sum Frequency Generation Spectra at Aqueous Interfaces. Chem. Rev. 2014, 114, 8447-8470.

30. Ohto, T.; Usui, K.; Hasegawa, T.; Bonn, M.; Nagata, Y. Toward ab initio molecular dynamics modeling for sum-frequency generation spectra; an efficient algorithm based on surface-specific velocity-velocity correlation function. J. Chem. Phys. 2015, 143, Art. No. 124702.

31. Khatib, R.; Backus, E. H. G.; Bonn, M.; Perez-Haro, M.-J.; Gaigeot, M.-P.; Sulpizi, M. Water orientation and hydrogen-bond structure at the fluorite/water interface. Scientific Reports 2016, 6, Art. No. 24287.

32. Khatib, R.; Sulpizi, M. Sum Frequency Generation Spectra from Velocity-Velocity Correlation Functions. J. Phys. Chem. Lett. 2017, 8, 1310-1314.

33. Valley, N. A.; Richmond, G. L. Computational Vibrational Sum Frequency Spectra of Formaldehyde and Hydroxymethanesulfonate at Aqueous Interfaces. J. Phys. Chem. C. 2016, $120,14122-14129$.

34. Ishiyama, T.; Takahashi, H.; Morita, A. Molecular dynamics simulations of surfacespecific bonding of the hydrogen network of water: A solution to the low sum-frequency spectra. Phys.Rev. B 2012, 86, 035408. 
35. Beierlein, F. R.; Krause, A. M.; Jäger, C. M.; Fita, P.; Vauthey, E.; Clark, T. Molecular Dynamics Simulations of Liquid Phase Interfaces: Understanding the Structure of the Glycerol/Water-Dodecane System. Langmuir 2013, 29, 11898-11907.

36. Sulpizi, M.; Salanne, M.; Sprik, M.; Gaigeot, M.-P. Vibrational Sum Frequency Generation Spectroscopy of the Water Liquid-Vapor Interface from Density Functional TheoryBased Molecular Dynamics Simulations. J. Phys. Chem. Lett. 2013, 4, 83-87.

37. Thomas, M.; Brehm, M.; Fligg, R.; Vöhringer, P.; Kirchner, B. Computing vibrational spectra from ab initio molecular dynamics. Phys. Chem. Chem. Phys. 2013, 15, 6608-6622.

38. Liang, C.; Jeon, J.; Cho, M. Ab initio modeling of the vibrational sum-frequency generation spectrum of interfacial water. J. Phys. Chem. Lett. 2019, 10, 1153-1158.

39. Ishiyama, T.; Takahashi, H.; Morita, A. Vibrational spectrum at a water surface: a hybrid quantum mechanics/molecular mechanics molecular dynamics approach. J. Phys.: Condens. Matt. 2012, 24, 124107.

40. Martins-Costa, M. T. C.; Ruiz-López, M. F. Highly accurate computation of free energies in complex systems through horsetail QM/MM molecular dynamics combined with free-energy perturbation theory. Theoret. Chem. Acc. 2017, 136, 50.

41. Bistafa, C.; Kitamura, Y.; Martins-Costa, M. T. C.; Nagaoka, M.; Ruiz-López, M. F. A Cost-Effective Method for Free-Energy Minimization in Complex Systems with Elaborated Ab Initio Potentials. J. Chem. Theor. Comp. 2018, 14, 3262-3271. 
42. Bistafa, C.; Kitamura, Y.; Martins-Costa, M. T. C.; Nagaoka, M.; Ruiz-Lopez, M. F. Vibrational Spectroscopy in Solution Through Perturbative Ab Initio Molecular Dynamics Simulations. J. Chem. Theor. Comput. 2019, 15, 4615-4622.

43. Matsumoto, M.; Takaoka, Y.; Kataoka, Y. Liquid vapor interface of water-methanol mixture .1. Computer-simulation. J. Chem. Phys. 1993, 98, 1464-1472.

44. Morita, A. Molecular dynamics study of mass accommodation of methanol at liquidvapor interfaces of methanol/water binary solutions of various concentrations. Chem. Phys. Lett. 2003, 375, 1-8.

45. Paul, S.; Chandra, A. Hydrogen bond properties and dynamics of liquid-vapor interfaces of aqueous methanol solutions. J. Chem. Theor. Comput. 2005, 1, 1221-1231.

46. Partay, L.; Jedlovszky, P.; Vincze, A.; Horvai, G. Structure of the liquid-vapor interface of water-methanol mixtures as seen from Monte Carlo Simulations. J. Phys. Chem. B 2005, 109, 20493-20503.

47. Chen, H.; Gan, W.; Lu, R.; Guo, Y.; Wang, H. F. Determination of structure and energetics for Gibbs surface adsorption layers of binary liquid mixture 2. Methanol plus water. $J$. Phys. Chem. B 2005, 109, 8064-8075.

48. Chang, T. M.; Dang, L. X. Liquid-vapor interface of methanol-water mixtures: A molecular dynamics study. J. Phys. Chem. B 2005, 109, 5759-5765.

49. Partay, L.; Jedlovszky, P.; Horvai, G. Temperature dependence of the structure of the liquid-vapour interface of aqueous methanol solutions. J. Mol. Liq. 2007, 134, 111-119. 
50. Partay, L. B.; Jedlovszky, P.; Vincze, A.; Horvai, G. Properties of free surface of water Methanol mixtures. Analysis of the truly interfacial molecular layer in computer simulation. $J$. Phys. Chem. B 2008, 112, 5428-5438.

51. Harper, K.; Minofar, B.; Sierra-Hernandez, M. R.; Casillas-Ituarte, N. N.; Roeselova, M.; Allen, H. C. Surface Residence and Uptake of Methyl Chloride and Methyl Alcohol at the Air/Water Interface Studied by Vibrational Sum Frequency Spectroscopy and Molecular Dynamics. J. Phys. Chem. A 2009, 113, 2015-2024.

52. Hub, J. S.; Caleman, C.; van der Spoel, D. Organic molecules on the surface of water droplets - an energetic perspective. Phys. Chem. Chem. Phys. 2012, 14, 9537-9545.

53. Martins-Costa, M. T. C.; Ruiz-López, M. F. Advances in QM/MM Molecular Dynamics Simulations of Chemical Processes at Aqueous Interfaces. In Quantum Modeling of Complex Molecular Systems, Rivail, J.-L.; Ruiz-Lopez, M. F.; Assfeld, X., Eds. Springer International Publishing: Cham, 2015; pp 303-324.

54. Superfine, R.; Huang, J.; Shen, Y. Nonlinear optical studies of the pure liquid/vapor interface: Vibrational spectra and polar ordering. Phys. Rev. Lett. 1991, 66, 1066-1069.

55. Wolfrum, K.; Graener, H.; Laubereau, A. Sum-frequency vibrational spectroscopy at the liquid air interface of methanol - water solutions. Chem. Phys. Lett. 1993, 213, 41-46.

56. Huang, J. Y.; Wu, M. H. Nonlinear-optical studies of binary-mixtures of hydrogenbonded liquids. Phys. Rev. E 1994, 50, 3737-3746. 
57. Stanners, C. D.; Du, Q.; Chin, R. P.; Cremer, P.; Somorjai, G. A.; Shen, Y. R. Polar ordering at the liquid-vapor interface of n-alcohols (C1-C8). Chem. Phys. Lett. 1995, 232, 407413.

58. Ma, G.; Allen, H. C. Surface studies of aqueous methanol solutions by vibrational broad bandwidth sum frequency generation spectroscopy. J. Phys. Chem. B 2003, 107, 6343-6349.

59. Sung, J. H.; Park, K.; Kim, D. Surfaces of alcohol-water mixtures studied by sumfrequency generation vibrational spectroscopy. J. Phys. Chem. B 2005, 109, 18507-18514.

60. Liu, W.-T.; Zhang, L.; Shen, Y. Interfacial structures of methanol: water mixtures at a hydrophobic interface probed by sum-frequency vibrational spectroscopy. J. Chem. Phys. 2006, 125, Art. No. 144711.

61. Ishihara, T.; Ishiyama, T.; Morita, A. Surface structure of methanol/water solutions via sum frequency orientational analysis and molecular dynamics simulation. J. Phys. Chem. C. 2015, 119, 9879-9889.

62. Li, X.; Deng, G.-H.; Feng, R.-J.; Lin, K.; Zhang, Z.; Bai, Y.; Lu, Z.; Guo, Y. Salt effect on molecular orientation at air/liquid methanol interface. Chin. Chem. Lett. 2016, 27, 535-539.

63. Li, X.; Liu, J. C.; Zhang, K.; Zhang, Y.; Zhang, Y. H.; Zheng, R. H.; Shi, Q.; Guo, Y.; Lu, Z. Toward Unraveling the Puzzle of Sum Frequency Generation Spectra at Interface of Aqueous Methanol Solution: Effects of Concentration-Dependent Hyperpolarizability. J. Phys. Chem. C 2019, 123, 12975-12983.

64. McQuarrie, D. A. Statistical Mechanics. Harper and Row: New York, 1976. 
65. Buchner, M.; Ladanyi, B. M.; Stratt, R. M. The short-time dynamics of molecular liquids. Instantaneous-normal-mode theory. J. Chem. Phys. 1992, 97, 8522-8535.

66. Stratt, R. M. THE INSTANTANEOUS NORMAL-MODES OF LIQUIDS. Acc. Chem.. Res. 1995, 28, 201-207.

67. Keyes, T. Instantaneous normal mode approach to liquid state dynamics. J. Phys. Chem. A 1997, 101, 2921-2930.

68. Murry, R. L.; Fourkas, J. T.; Li, W. X.; Keyes, T. Molecular coordinates for instantaneous normal mode calculations. I. Coordinate dependence. J. Chem. Phys. 1999, 110, 10410-10422.

69. Perry, A.; Ahlborn, H.; Space, B.; Moore, P. B. A combined time correlation function and instantaneous normal mode study of the sum frequency generation spectroscopy of the water/vapor interface. J. Chem. Phys. 2003, 118, 8411-8419.

70. Perry, A.; Neipert, C.; Kasprzyk, C. R.; Green, T.; Space, B.; Moore, P. B. A theoretical description of the polarization dependence of the sum frequency generation spectroscopy of the water/vapor interface. J. Chem. Phys. 2005, 123, Art. No. 144705.

71. Kalbfleisch, T.; Keyes, T. Untangling the physical contributions to instantaneous normal mode approximations: Inhomogeneous broadening, motional narrowing, and energy relaxation. J. Chem. Phys. 1998, 108, 7375-7383.

72. Schmitz, M.; Tavan, P. Vibrational spectra from atomic fluctuations in dynamics simulations. I. Theory, limitations, and a sample application. J. Chem. Phys. 2004, 121, 1223312246. 
73. Schmitz, M.; Tavan, P. Vibrational spectra from atomic fluctuations in dynamics simulations. II. Solvent-induced frequency fluctuations at femtosecond time resolution. J. Chem. Phys. 2004, 121, 12247-12258.

74. Nonella, M.; Mathias, G.; Tavan, P. Infrared spectrum of p-benzoquinone in water obtained from a QM/MM hybrid molecular dynamics simulation. J. Phys. Chem. A 2003, 107, $8638-8647$.

75. Cui, Q.; Karplus, M. Molecular properties from combined QM/MM methods. I. Analytical second derivative and vibrational calculations. J. Chem. Phys. 2000, 112, 1133-1149.

76. Kitamura, Y.; Takenaka, N.; Koyano, Y.; Nagaoka, M. Dual Approach to Vibrational Spectra in Solution: Microscopic Influence of Hydrogen Bonding to the State of Motion of Glycine in Water. J. Chem. Theor. Comput. 2014, 10, 3369-3379.

77. Okuyama-Yoshida, N.; Nagaoka, M.; Yamabe, T. Transition-state optimization on free energy surface: Toward solution chemical reaction ergodography. Int. J. Quantum Chem. 1998, $70,95-103$.

78. Okuyama-Yoshida, N.; Kataoka, K.; Nagaoka, M.; Yamabe, T. Structure optimization via free energy gradient method: Application to glycine zwitterion in aqueous solution. J. Chem. Phys. 2000, 113, 3519-3524.

79. Hirao, H.; Nagae, Y.; Nagaoka, M. Transition-state optimization by the free energy gradient method: Application to aqueous-phase Menshutkin reaction between ammonia and methyl chloride. Chem. Phys. Lett. 2001, 348, 350-356. 
80. Nagaoka, M. Structure optimization of solute molecules via free energy gradient method. Bull. Korean Chem. Soc. 2003, 24, 805-808.

81. Chipot, C.; Pohorille, A. Free energy calculations. Springer-Verlag: Berlin Heidelberg, 2007.

82. Retegan, M.; Martins-Costa, M.; Ruiz-López, M. F. Free energy calculations using duallevel Born-Oppenheimer molecular dynamics. J. Chem. Phys. 2010, 133, Art. No. 064103.

83. Becke, A. D. Density-Functional thermochemistry. iii. The role of exact exchange. $J$. Chem. Phys. 1993, 98, 5648-5652.

84. Hehre, W. J.; Ditchfield, R.; Pople, J. A. Self-Consistent Molecular Orbital Methods. XII. Further Extensions of Gaussian-Type Basis Sets for Use in Molecular Orbital Studies of Organic Molecules. J. Chem. Phys. 1972, 56, 2257-2261.

85. Hehre, W. J.; Stewart, R. F.; Pople, J. A. Self-consistent molecular orbital methods. I Use of gaussian expansions of Slater-type atomic orbitals. J. Chem. Phys. 1969, 51, 2657-2664.

86. Krishnan, R.; Binkley, J. S.; Seeger, R.; Pople, J. A. Self-Consistent Molecular-Orbital Methods .20. Basis Set For Correlated Wave-Functions. J. Chem. Phys. 1980, 72, 650-654.

87. Jorgensen, W. L.; Chandrashekar, J.; Madura, J. D.; Impey, W. R.; Klein, M. L. Comparison of simple potential functions for simulating liquid water. J. Chem. Phys. 1983, 79, 926-935.

88. Dang, L. X.; Pettitt, B. M. Simple intramolecular model potentials for water. J. Phys. Chem. 1987, 91, 3349-3354. 
89. Feugmo, C. G. T.; Liegeois, V.; Champagne, B. Coupled-cluster sum-frequency generation nonlinear susceptibilities of methyl $(\mathrm{CH} 3)$ and methylene $(\mathrm{CH} 2)$ groups. Phys. Chem. Chem. Phys. 2017, 19, 29822-29832.

90. Jorgensen, W. L.; Tirado-Rives, J. The OPLS potential functions for proteins, energy minimizations for crystals of cyclic peptides and crambin. J. Am. Chem. Soc. 1988, 110, 16571666.

91. Hoover, W. G. Canonical dynamics: Equilibrium phase-space distributions. Phys. Rev. A 1985, 31, 1695-1697.

92. Nosé, S. A unified formulation of the constant temperature molecular dynamics methods. J. Chem. Phys. 1984, 81, 511-519.

93. Frisch, M. J.; Trucks, G. W.; Schlegel, H. B.; Scuseria, G. E.; Robb, M. A.; Cheeseman, J. R.; Scalmani, G.; Barone, V.; Mennucci, B.; Petersson, G. A., et al. Gaussian 09, Gaussian, Inc.: Wallingford, CT, USA, 2009.

94. Ponder, J. W. TINKER: Software Tools for Molecular Design 4.2; Washington University School of Medicine: Saint Louis, MO.: 2004.

95. Martins-Costa, M. T. C.; Ruiz-López, M. F. Molecular dynamics of hydrogen peroxide in liquid water using a combined quantum/classical force field. Chem. Phys. 2007, 332, 341-347.

96. Yu, Y. Q.; Wang, Y. X.; Lin, K.; Hu, N. Y.; Zhou, X. G.; Liu, S. L. Complete Raman Spectral Assignment of Methanol in the C-H Stretching Region. J. Phys. Chem. A 2013, 117, $4377-4384$. 
97. Serrallach, A.; Meyer, R.; Günthard, H. H. Methanol and deuterated species: Infrared data, valence force field, rotamers, and conformation. J. Mol. Spectrosc. 1974, 52, 94-129.

98. Schwartz, M.; Moradi-Araghi, A.; Koehler, W. H. Fermi resonance in aqueous methanol. J. Mol. Struct. 1980, 63, 279-285.

99. Schwartz, M.; Moradi-Araghi, A.; Koehler, W. H. Solvent and temperature dependence of the fermi resonance parameters in methanol. J. Mol. Struct. 1982, 81, 245-252.

100. Ishiyama, T.; Sokolov, V. V.; Morita, A. Molecular dynamics simulation of liquid methanol. II. Unified assignment of infrared, raman, and sum frequency generation vibrational spectra in methyl C-H stretching region. J. Chem. Phys. 2011, 134, Art. No. 024510.

101. Carr, J. K.; Wang, L.; Roy, S.; Skinner, J. L. Theoretical Sum Frequency Generation Spectroscopy of Peptides. J. Phys. Chem. B 2015, 119, 8969-8983.

102. Kim, J.; Chou, K. C.; Somorjai, G. A. Structure and dynamics of acetonitrile at the air/liquid interface of binary solutions studied by infrared-visible sum frequency generation. $J$. Phys. Chem. B 2003, 107, 1592-1596.

103. Saito, K.; Peng, Q. L.; Qiao, L.; Wang, L.; Joutsuka, T.; Ishiyama, T.; Ye, S.; Morita, A. Theoretical and experimental examination of SFG polarization analysis at acetonitrile-water solution surfaces. Phys. Chem. Chem. Phys. 2017, 19, 8941-8961. 